\title{
Dietary Management of a Patient with Both Maple Syrup Urine Disease and Type I Diabetes
}

\author{
๑ Mehmet Gündüz1, ๑ Nevra Koç1, ๑ Özlem Ünal1, ๑ Seyit Ahmet Uçaktürk2 \\ 1 University of Health Sciences, Ankara Children Health and Diseases Hematology Oncology Training and Research Hospital, Clinic of Pediatric \\ Nutrition and Metabolism, Ankara, Turkey \\ 2 University of Health Sciences, Ankara Children Health and Diseases Hematology Oncology Training and Research Hospital, Clinic of Pediatric \\ Endocrinology and Metabolism, Ankara, Turkey
}

\begin{abstract}
Maple Syrup Urine disease (MSUD) is caused by the deficiency of the branched chain 2-ketoacid dehydrogenase complex. Type I diabetes mellitus (TIDM) is a chronic illness characterized by the body's inability to produce insulin due to the autoimmune destruction of the beta cells in the pancreas. A case with both MSUD and TIDM has not been reported previously. In this study, we presented a patient with both MSUD and TIDM, dietary management and the follow up period. Dietary management could be difficult in the presence of two disorders affecting more than one macronutrient component such as both carbohydrate and protein. Our patient had good metabolic control in the followup period. Treatment was successful with intensive insulin, frequent feeding and a leucine restricted diet.

Keywords: Maple Syrup Urine disease, Type I diabetes mellitus, diet, children, metabolic control
\end{abstract}

\section{Introduction}

Maple Syrup Urine disease (MSUD) is caused by the deficiency of the branched chain 2-ketoacid dehydrogenase (BCKD) complex, the second common step in the catabolism of the three branched chain amino acids (BCAA), leucine, isoleucine and valine. Long term dietary treatment is aimed at reducing the accumulation of toxic metabolites, while at the same time maintaining normal physical development and nutritional status, and preventing catabolism. Type I diabetes mellitus (TIDM) is a chronic illness characterized by the body's inability to produce insulin due to the autoimmune destruction of the beta cells in the pancreas. Patients with TIDM need lifelong insulin therapy. One of the first steps in managing TIDM is dietary control. All patients on insulin should have a comprehensive diet plan, including a daily calorie intake prescription, recommendations for amounts of dietary carbohydrate, fat, and protein, and instructions on how to divide calories between meals and snacks. A case with both MSUD and TIDM has not been hitherto reported. Here, we present a patient with both MSUD and TIDM, and the dietary management of the patient.

\section{Case Report}

A four-year-old boy who was diagnosed with classical MSUD in the newborn period was admitted to our hospital with lethargy, polyuria and polydipsia. He had mild spasticity in the limbs and mild axial hypotonia as a sequel of a metabolic crisis attack in the newborn period. He had been found carrying homozygous c.757G>A; p.Ala253Thr mutation. At his last admission, his blood glucose level was $665 \mathrm{mg} / \mathrm{dL}$. He had metabolic acidosis, and ketosis. Laboratory findings were: $\mathrm{pH}: 6.9, \mathrm{pO}_{2}: 117 \mathrm{mmHg}, \mathrm{pCO}_{2}: 29.7 \mathrm{mmHg}, \mathrm{HCO}_{3}$ : 6.5, blood ketone level: $6.2 \mathrm{mmol} / \mathrm{L}$. The hemoglobin A1c level was $11.2 \%$. C-peptide level was low $(0.486 \mathrm{ng} / \mathrm{mL}$, 
normal range 0.9-7.1). Diabetes related autoantibodies were found positive and were as follows; islet cell antibody: $1: 10(n<1: 10)$, anti-insuline antibody: $0.60 \%(n<0.40)$, antiglutamic acid decarboxylase: $647.57 \mathrm{IU} / \mathrm{mL}(\mathrm{n}<10)$. Leucine level was $451 \mu \mathrm{mol} / \mathrm{L}(\mathrm{n}=55-164)$. Ketoacidosis was treated with intravenous regular insulin and fluid. Leucine free amino acid mixture was used for snacks until blood glucose levels were regulated. Following the recovery period, intensive insulin (four dosages a day) treatment was started. Three main meals and three snacks were commenced. The dietary plan of the patient consisted of $2 \mathrm{~g} / \mathrm{kg} /$ day protein, $100 \mathrm{kcal} / \mathrm{kg}$ energy, and $30 \mathrm{mg} / \mathrm{kg} /$ day leucine. His dietary schema is shown in Figure 1. He is still going on with this diet along with thiamine treatment, and doing well. In the 1.5 year-period after the additional TIDM diagnosis, he has not experienced a metabolic crisis associated with TIDM or MSUD. He has only a mild global developmental delay.

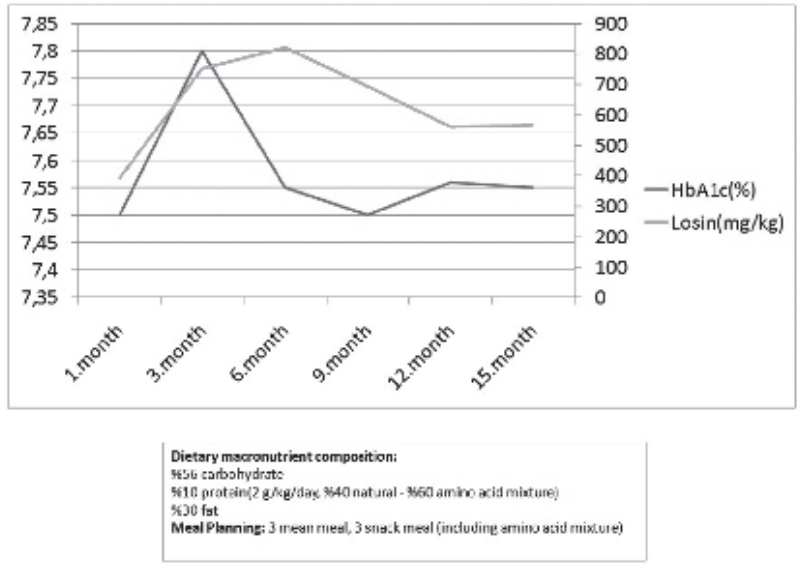

Figure 1. Clinical findings and dietary management of patient

\section{Discussion}

Here we presented a case with both MSUD and TIDM together with our dietary management approach. MSUD, also known as branched-chain ketoaciduria, is a disorder affecting the BCAA. MSUD occurs in approximately 1 in 86.800 to 185.000 live births $(1,2)$. TIDM is a heterogeneous disorder characterized by the destruction of pancreatic beta cells, culminating in absolute insulin deficiency. Data from large epidemiologic studies worldwide indicate that the incidence of TIDM has been increasing by $2-5 \%$ worldwide and that the prevalence of TIDM is approximately 1 in 300 in the US by 18 years of age (3). Research on risk factors for TIDM is an active area of research to identify genetic and environmental triggers that could potentially be targeted for intervention. BCAA, which accumulates in Type II diabetes, contributes to insulin resistance. The activity of the BCKD complex, the rate-limiting enzyme in the BCAA catabolism, has clearly diminished. Therefore, diabetes contributes to the increase in BCAA. Lian et al. (4) demonstrated that mitochondrial phosphatase $2 \mathrm{C}$ (PP2Cm), which is associated with increased BCKD activity, reduced in hippocampal mice with Type II diabetes. Adiponectin (APN) is the new regulator of $\mathrm{PP} 2 \mathrm{Cm}$ and BCAA. Targeting APN pharmacologically suggests that the catabolism of BCAA may be ameliorated. Our patient had TIDM, and diabetes related autoantibodies were demonstrated. Demonstration of the mechanism underlying the presence of two disorders together, MSUD and TIDM could be accepted as coincidence in this patient. Although he was treated with intensive insulin regimen, accumulation of BCAA may contribute to the increase of insulin in the follow-up period. Experimental treatments such as mitochondrial PP2Cm could be considered as a promising agent in the future. Exogenous (dietary) BCAA are major precursors for protein synthesis. Normally, they are also used as an alternative energy source when consumed in excess for anabolic needs or during endogenous muscle protein catabolism. The goals of medical nutrition therapy in MSUD are to rapidly reduce toxic metabolites by restricting dietary BCAA to amounts allowing individuals to achieve and maintain plasma BCAA amino acid concentrations within the targeted treatment ranges; reduce catabolism; promote anabolism; monitor nutritional status and alter intake to promote normal growth, development and health maintenance; evaluate thiamin responsiveness if the individual has residual BCKD activity; and supplement with thiamin if the individual is responsive (5). According to the Nutrition Management Guideline published by Frazier et al. (5), the recommended dietary requirements for children with MSUD were as follows; 35-65 mg/kg leucine, 20-30 mg/ $\mathrm{kg}$ isoleucine, $30-50 \mathrm{mg} / \mathrm{kg}$ valine, $1.3-2.0 \mathrm{~g} / \mathrm{kg}$ protein, and $50-120 \mathrm{kcal} / \mathrm{kg}$ energy. Our patient's dietary content was compatible with this guideline. Although median leucine level was slightly higher than the recommended levels, there was no metabolic disturbance and crisis other than the first metabolic crisis in the newborn period and during diabetic ketoacidosis attack. Significant advances have been made in the clinical care of TIDM. A number of therapeutic options for persons with TIDM currently exist, which include multiple daily injections of rapid acting insulin with meals combined with a daily basal insulin. In risky situations such as severe infections and occurrence of ketosis, high carbohydrate intake could be commenced with increased insulin doses.

In summary, dietary management could be difficult the presence of two disorders affecting more than one macronutrient component such as both carbohydrate and protein. Our patient had good metabolic control in the followup period. Successful treatment was achieved with intensive insulin, frequent feeding and a leucine restricted diet.

\section{Ethics}

Informed Consent: It was taken from the mother. Peer-review: External and internal peer-reviewed.

\section{Authorship Contributions}

Surgicaland Medical Practices: M.G., N.K., Ö.Ü., S.A.U., Concept: M.G., N.K., Design: M.G., N.K., Ö.Ü., Data Collection 
or Processing: N.K., S.A.U., Analysis or Interpretation: N.K., Literature Search: N.K., Writing: N.K.

Conflict of Interest: No conflict of interest was declared by the authors.

Financial Disclosure: The authors declared that this study received no financial support.

\section{References}

1. Chuang DT, Shih VE. Maple syrup urine disease (branchedchain ketoaciduria). In: The metabolic and molecular bases of inherited disease, 8th ed, Scriver $C R$, Beaudet $A L$, Sly WS, Valle D (Eds), McGraw-Hill, New York 2001.p.1971.
2. Quental S, Vilarinho $L$, Martins $E$, et al. Incidence of maple syrup urine disease in Portugal. Mol Genet Metab 2010;100:385-7.

3. Maahs DM, West NA, Lawrence JM, et al. Chapter 1: Epidemiology of Type 1 Diabetes. Endocrinol Metab Clin North Am 2010;39:481-97.

4. Lian K, Du C, Liu Y, et al. Impaired adiponectin signaling contributes to disturbed catabolism of branched-chain amino acids in diabetic mice. Diabetes 2015;64:49-59.

5. Frazier DM, Allgeier $\mathrm{C}$, Homer $\mathrm{C}$, et al. Nutrition management guideline for maple syrup urine disease: an evidenceand consensus-based approach. Mol Genet Metab 2014;112:210-7. 